\title{
A NOVEL, INTEGRATED TREATMENT SYSTEM FOR COAL WASTEWATERS:
}

\author{
QUARTERLY REPORT:
}

FOR THE PERIOD September 2, 1992 - December 1, 1992.

PIs:

Prof. Henry Y. Wang

Department of Chemical Engineering

The University of Michigan

Ann Artor, MI 48109-2136.

DOE /PC/91295--T5

DE93 011632

$\&$

Dr. Keeran R. Srinivasan

Assoc. Research Scientist

Department of Chemical Engineering

The University of Michigan

Ann Arbor, MI 48109-2136.

Submitted To:

Pittsburgh Energy Technology Center

U. S. Department of Energy

Pittsburgh, PA.

\begin{abstract}
:
The aims of this study are to develop, characterize and optimize a novel treatment scheme that would be effective simultaneously against the toxic organics and the heavy metals present in coal conversion wastewaters. Hec-CBDA-DT, (HCDT), a modified hectorite containing a mixed bilayer of a cationic (CBDA) and a diamine (DT) type surfactants, has been shown to adsorb $\mathrm{Cr}$ (VI) strongly at pH below 5.0. A second kind of a modified clay, montmorillonite-DT, (MONT-DT), in which the protonated form of the diamine is directly attached to the negative sites of clay surface, has also been found to adsorb $\operatorname{Cr}(\mathrm{VI})$ as strongly as Hec-CBDA-DT at a pH of 4.5 .
\end{abstract}

The adsorption of $\mathrm{Cr}$ (VI) onto these two modified-clays is strongly inhibited by an increase in the ionic strength of the medium. It is inferred that the main mechanism of adsorption of $\mathrm{Cr}(\mathrm{VI})$ onto these two clay complexes is of the counterion type.

Preliminary data on the partitioning of phenanthrene, a three-ringed polyaromatic hydrocarbon (PAH), reveal that organic carbon located on the external surfaces of clay complexes is more effective in partitioning nonionic, toxic organic compounds.

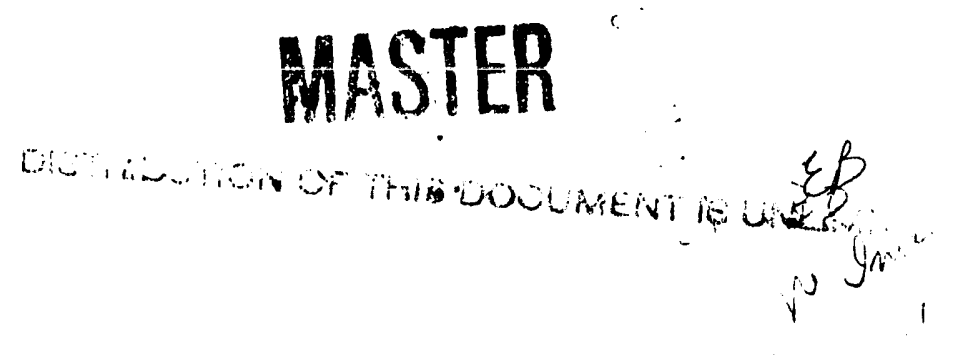


DE-FG22-91PC91295

\begin{abstract}
A NOVEL, INTEGRATED TREATMENT SYSTEM FOR COAL WASTEWATERS:
\end{abstract}

QUARTERLY REPORT:

FOR THE PERIOD September 2, 1992 - December 1, 1992.
PIs: $\quad$ Prof. Henry Y. Wang
Department of Chemical Engineering
The University of Michigan
Ann Arbor, MI 48109-2136.
\&
Dr. Keeran R. Srinivasan
Assoc. Research Scientist
Department of Chemical Engineering
The University of Michigan
Ann Arbor, MI 48109-2136.
Submitted To:
Pittsburgh Energy Technology Center
U. S. Department of Energy
Pittsburgh, PA.

\begin{abstract}
:
The aims of this study are to develop, characterize and optimize a novel treatment scheme that would be effective simultaneously against the toxic organics and the heavy metals present in coal conversion wastewaters. Hec-CBDA-DT, (HCDT), a modified hectorite containing a mixed bilayer of a cationic (CBDA) and a ciamine (DT) type surfactants, has been shown to adsorb $\mathrm{Cr}$ (VI) strongly at pH below 5.0. A second kind of a modified clay, montmorillonite-DT, (MONT-DT), in which the protonated form of the diamine is directly attached to the negative sites of clay surface, has also been found to adsorb $\mathrm{Cr}(\mathrm{VI})$ as strongly as Hec-CBDA-DT at a pH of 4.5 .
\end{abstract}

The adsorption of $\mathrm{Cr}$ (VI) onto these two modified-clays is strongly inhibited by an increase in the ionic strength of the medium. It is inferred that th" main mechanism of adsorption of $\mathrm{Cr}(\mathrm{VI})$ onto these two clay complexes is of the crinterion type.

Preliminary data on the partitioning of phenanthrene, a three-ringed polyaromatic hydrocarbon (PAH), reveal that organic carbon located on the external surfaces of clay complexes is more effective in partitioning nonionic, toxic organic compounds. 
Fig. 1 Adsorption of $\mathrm{Cr}$ (VI) on Modified-Clays: $\mathrm{pH}$ Effect

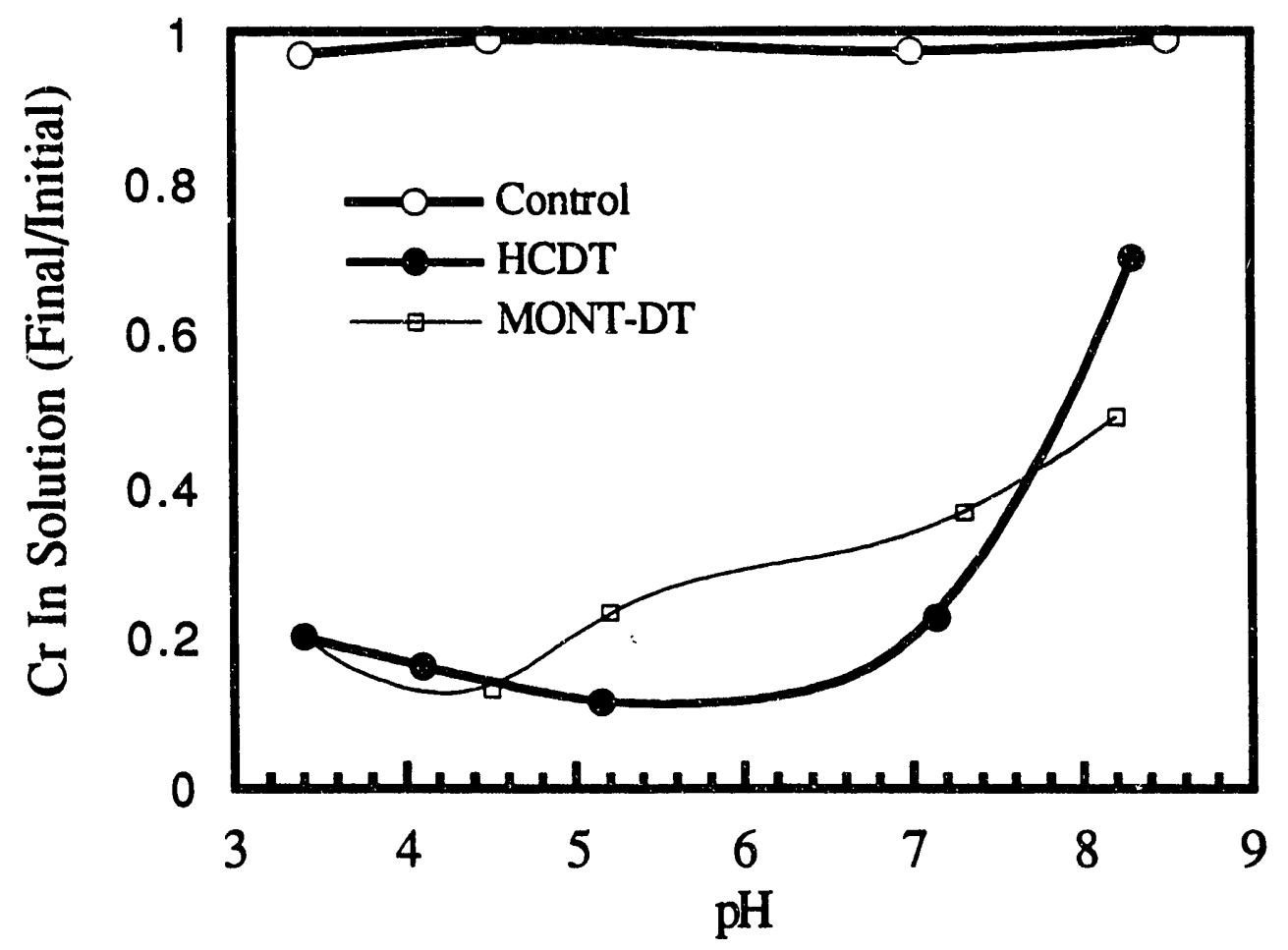

Fig. 2 Adsorption of $\mathrm{Cr}(\mathrm{VI})$ onto Modified-Clays: pH Effect

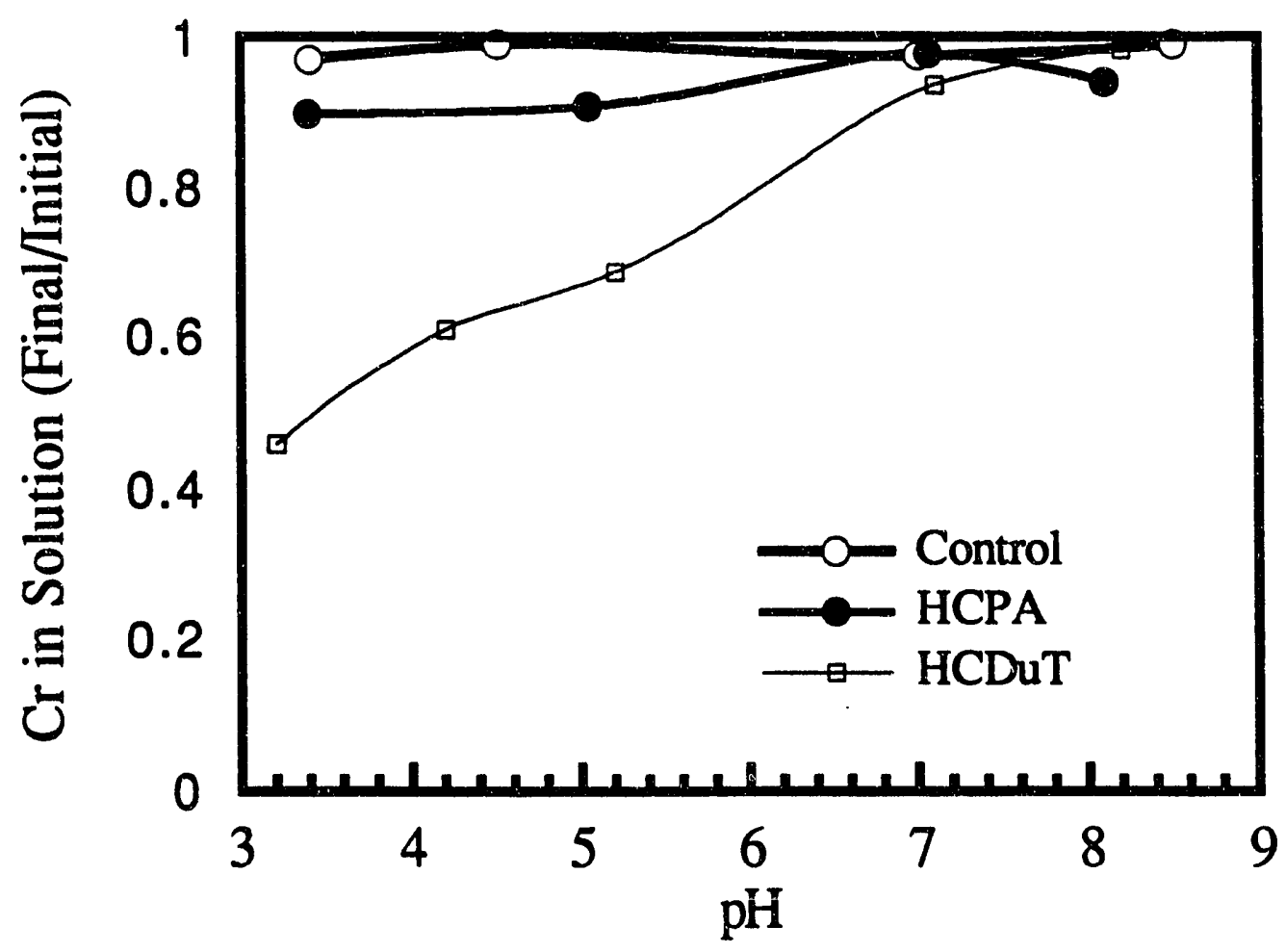


Fig. 3 Adsorption of $\mathrm{Cr}(\mathrm{VI})$ onto HCDT and MONT-DT at $\mathrm{pH} 4.5$

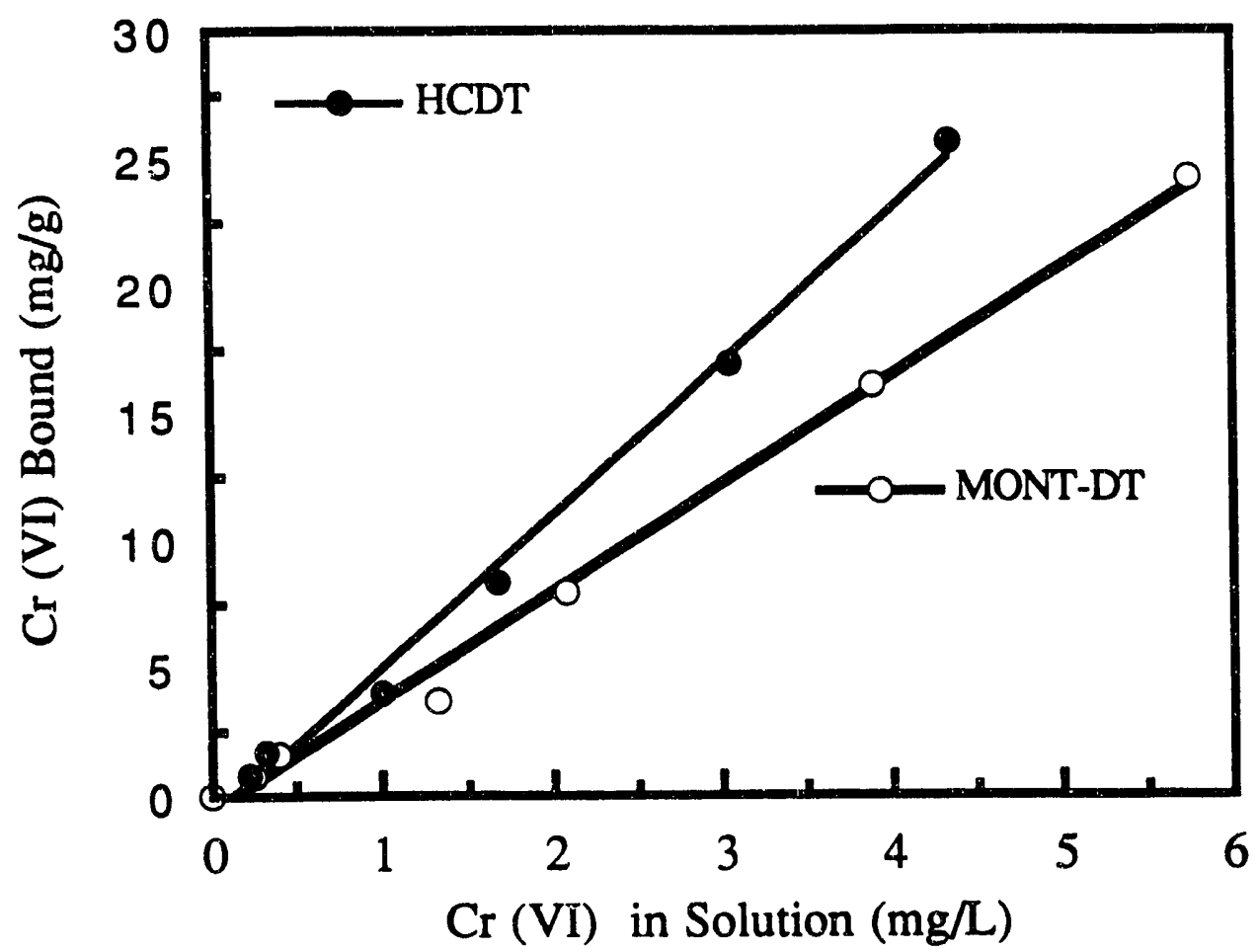

Fig. 4 Adsorption of $\mathrm{Cr}(\mathrm{VI})$ on Modified-Clays at $\mathrm{pH}$ 4.5: Ionic Strength Effect

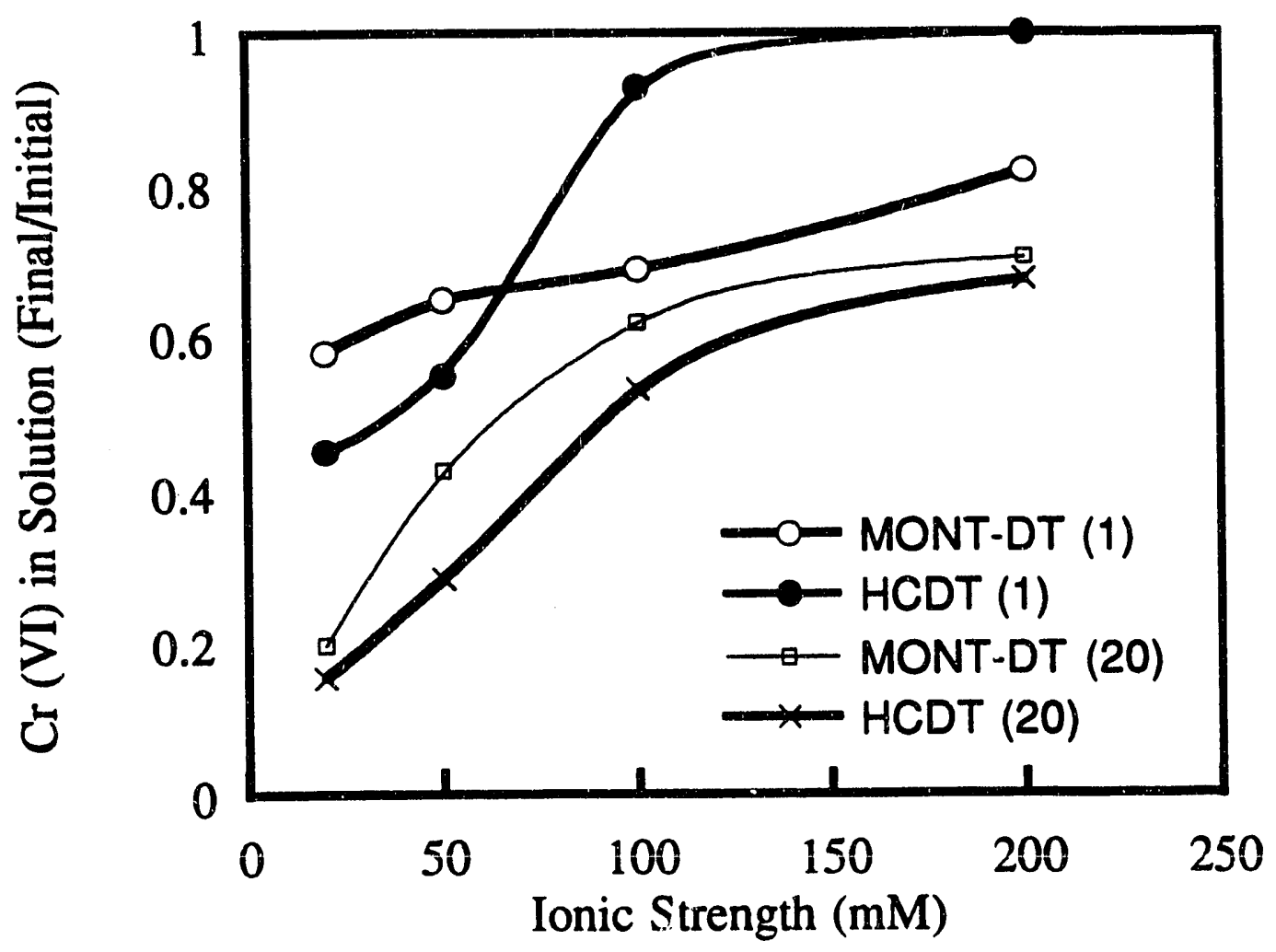


6

Fig. 5 Adsorption of $\mathrm{Cr}(\mathrm{VI})$ onto Modified-Clays at $\mathrm{pH}$ 4.5: Ionic Strength $50 \mathrm{mM}$

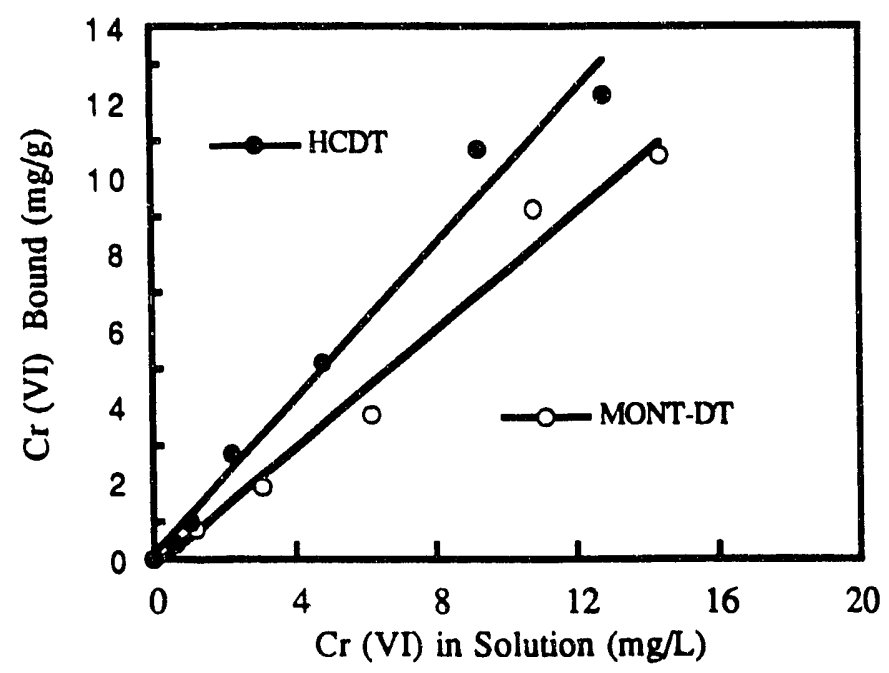

Fig. 6 Adsorption of $\mathrm{Cr}$ (VI) at $\mathrm{pH}$ 4.5: Ionic Strength $200 \mathrm{mM}$

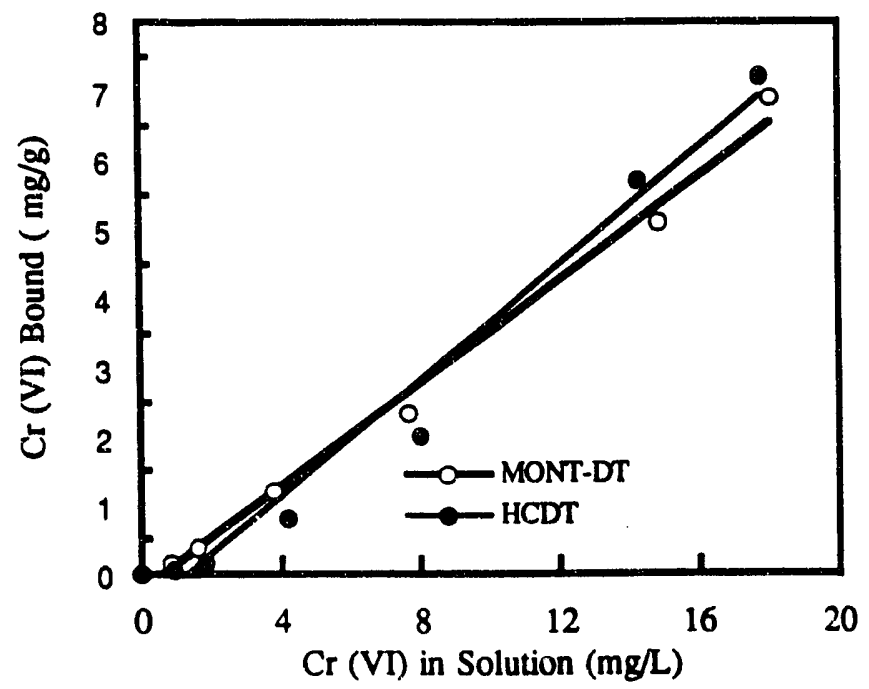

Fig. 7 Adsorption of Phenanthrene onto HCDT and MONT-DT

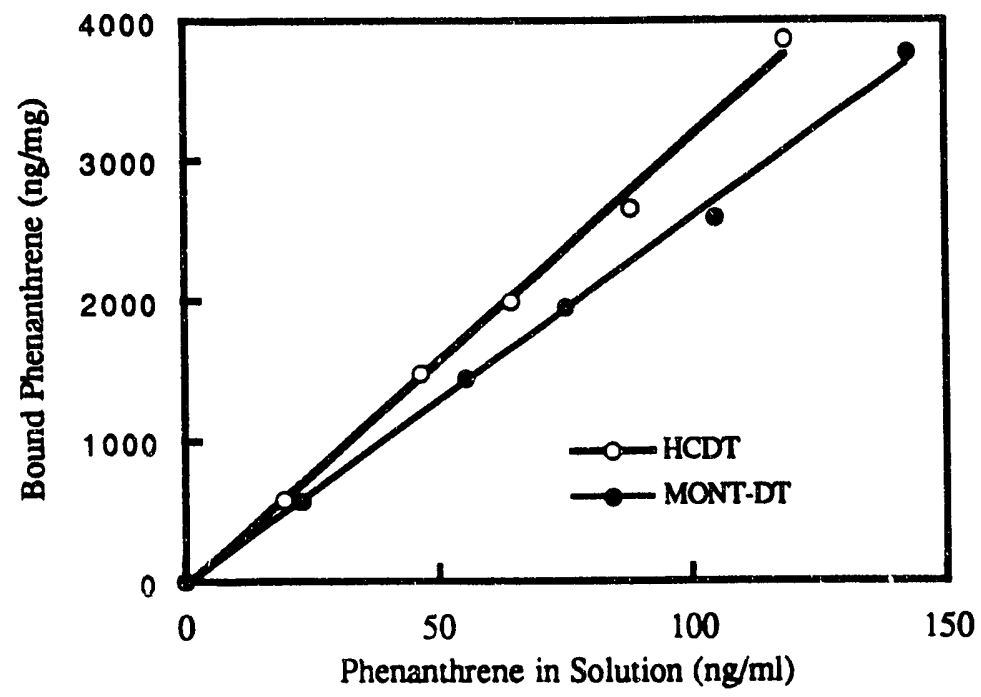


(5) Akzo Chemie America: Bulletin 85-1, "Specification and Properties of DUOMEEN Diamines and Diamine Salts", An Armak Chemical Publication, (1985).

(6) Susan K. Siegel and Dennis A. Clifford: U. S. EPA Publication. EPA/600/S288/007, April, 1988.

(7) Keeran R. Srinivasan and H. Scott Fogler: "Use of Inorgano-organo-Clays in the Treatment of Coal Conversion Wastewaters " Proc. 1990 International Symposium on Dioxin and Other Pollutants, held at Bayereuth, Fed. Republic of Germany, September, 1990.

\section{PRESENTATIONS:}

(1) On the Use of Modified-Clays for the Removal and Recovery of Heavy Metals From Wastewaters:

Keeran R. Srinivasan, Mike Moore, and Henry Y. Wang

The Annual Clay Minerals Society Meeting, Minneapolis, MN, November 1 - 5, 1992. 

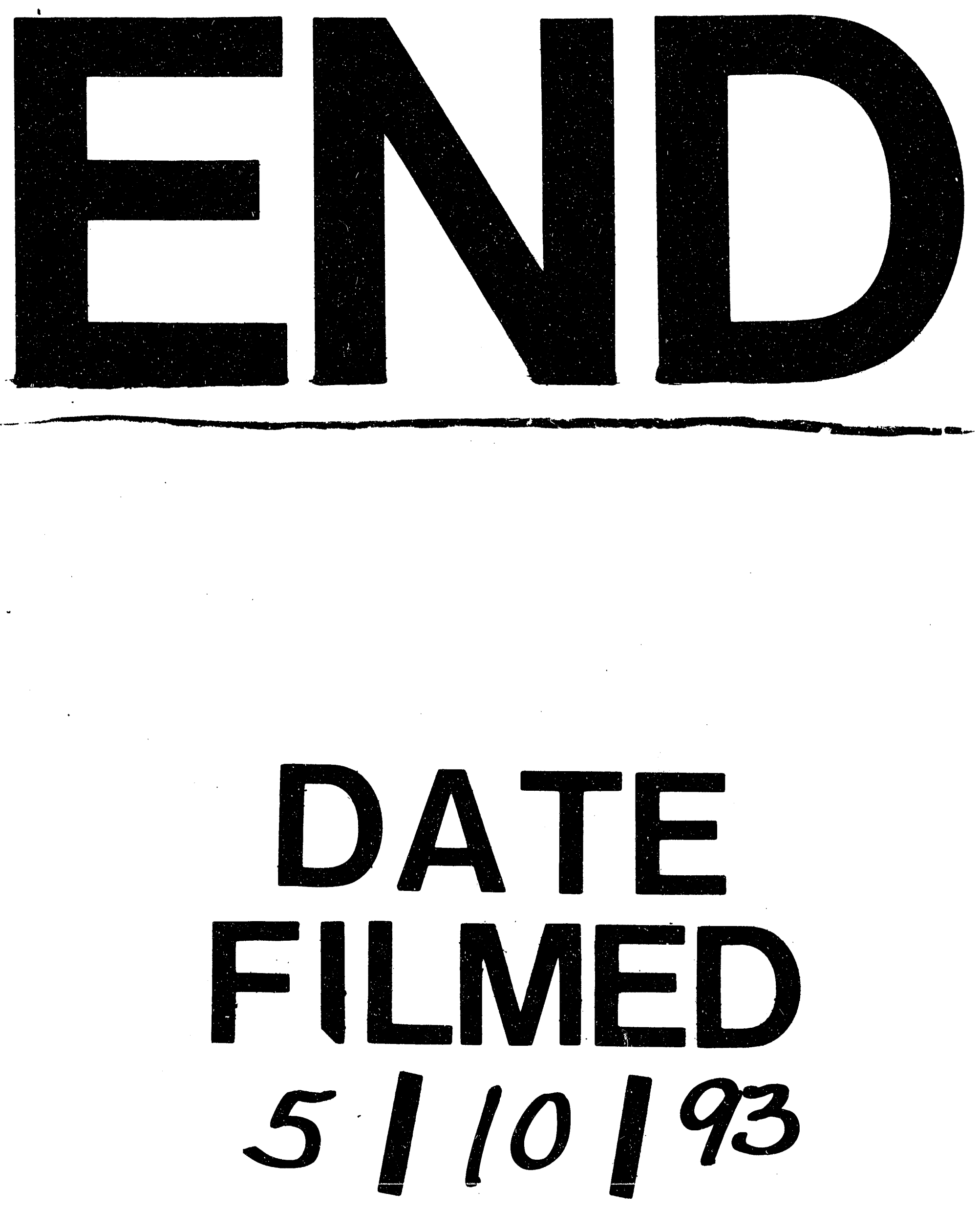
1 